\title{
solid

\section{Correlation effects in a one-dimensional electron gas with short-range interaction}

\author{
E. Demirel, B. Tanatar \\ Department of Physics, Bilkent University, Bilkent, 06533 Ankara, Turkey \\ Received 16 December 1998; accepted 21 December 1998 by R.T. Phillips
}

\begin{abstract}
We study the correlation effects in a one-dimensional electron gas with repulsive delta-function interaction. The correlation effects are described by a local-field correction which takes into account the short-range correlations. We find that the ground state energy is in good agreement with the exact result up to intermediate coupling strengths, showing an improvement over the STLS approximation. The compressibility, the static structure factor and the pair-correlation function are also calculated within the present approximation. (C) 1999 Elsevier Science Ltd. All rights reserved.
\end{abstract}

Keywords: A. Nanostructures; B. Electron-electron interactions; B. Thermodynamic properties

\section{Introduction}

One-dimensional (1D) electron systems are attracting a lot of interest [1-3], because of their applicability to naturally occurring organic conductors, artificially fabricated semiconductor structures, and certain materials exhibiting superconductivity. The study of many-body exchange and correlation effects in homogeneous quantum electron liquids is a field of active research and is of continuing interest because of the current applications. The random-phase approximation [4] (RPA) has been very successful in describing the dielectric properties of the interacting electron system with long-range Coulomb interaction in the high density limit. As the density of the electrons is lowered, the exchange and correlation effects become increasingly important. The Singwi, Tosi, Land and Sjölander (STLS) approach [5] is a powerful approximating theory to study the correlations in interacting electron systems in terms of the local-field factors.

One-dimensional fermions interacting via a repulsive $\delta$ function potential were treated by Yang [6] using the Bethe ansatz method. Friesen and Bergersen [7] numerically solved Yang's equations [6] to calculate the ground-state energy of the system, and compared them with the STLS results. Gold [810] studied the exchange-correlation effects in a $1 D$ electron gas with short-range interaction using the concept of local-field corrections. Gold [8] used an analytical expression for the static structure factor (SSF) with the STLS approach and compared his results with the STLS approach, where numerical results for the SSF are used. The comparison shows that the analytical form of the SSF is a good approximation of the real SSF. In this work we revisit the problem of $1 \mathrm{D}$ fermions interacting via a delta-function potential within the local field correction approach. We use the analytical form of the SSF which is employed by Gold [8]. We describe the correlation effects in the interacting electron gas in terms of a local-field factor introduced by Vashishta and Singwi [11] in an approximate way. We find that 
the exact ground-state energy [6] obtained by Friesen and Bergersen [7] is reproduced up to large values of the coupling constant. This is an improvement over the STLS scheme which show agreement with the exact ground state energy for small values of the coupling strength. Our calculations are motivated by similar improvements for the 1D interacting boson system, when the VS approach is used [12].

\section{Model and Theory}

We consider a system of electrons in 1D interacting via a contact potential $V\left(r_{1}, r_{2}\right)=V_{0} \delta\left(r_{1}-r_{2}\right)$, where $V_{0}$ is the interaction strength. In terms of the electron mass $m$ and the density of the particles $n$, we use the dimensionless parameter $\gamma=m V_{0} / n$ to characterize the strength of the coupling (we take $\hbar=1$ ).

Within the STLS approach, the LFC for 1D electron system is given by

$G_{\mathrm{STLS}}(\gamma)=\frac{1}{n \pi} \int_{0}^{\infty} d q[1-S(q)]$

which is independent of the wave vector variable. Using the mean-spherical approximation [13-16] (MSA), we can write the analytical expression for static structure factor

$S(q)=\left[\frac{1}{S_{0}^{2}}+\frac{4 n^{2}}{q^{2}} \gamma[1-G(\gamma)]\right]^{-1 / 2}$

$S_{0}(q)$ is the SSF of the noninteracting electron gas in $1 \mathrm{D}$, i.e. $S_{0}(q)=q / 2 k_{\mathrm{F}}$, for $q<2 k_{\mathrm{F}}$, and $S_{0}(q)=1$, for $q>2 k_{\mathrm{F}}$. The factor containing $\gamma[1-G(\gamma)]$ represents the contribution of the collective modes to the SSF. A cubic equation

$G(\gamma)=\frac{1+8 \gamma[1-G(\gamma)] / \pi^{2}}{2\left[1+4 \gamma[1-G(\gamma)] / \pi^{2}\right]^{1 / 2}}$

is obtained for the LFC [8].

In this work we choose a different local-field factor in the description of the ground state correlation effects for 1D electrons. We use the approach introduced by Vashishta and Singwi [11] (VS) which was originally constructed to satisfy the compressibility sum-rule. In the modified theory of Vashishta and Singwi $[9,10]$ the equilibrium pair correlation function $g(r)$ which enters the ansatz for the two-particle distribution function is amended by a correction term involving the density derivative of $g(r)$. For a onedimensional system of fermions interacting via a constant potential the local-field factor in the VS approximation now reads

$G_{\mathrm{VS}}(\gamma)=\left(1+a n \frac{\partial}{\partial n}\right) \frac{1}{n \pi} \int_{0}^{\infty} d q[1-S(q)]$,

where $a$ is an adjustable parameter. Note that the local-field factor $G$ is still independent of the wave vector variable as in the STLS approximation. As in the case of the STLS scheme, the above equation for $G$ has to solved self-consistently along with the SSF. This yields a highly nonlinear differential equation for $G_{\mathrm{VS}}(\gamma)$. Rather than attempting to solve the nonlinear differential equation numerically, we adopt a simpler approximation given by $G_{\mathrm{VS}}(\gamma)=\left(1-a \gamma \frac{\partial}{\partial \gamma}\right) G_{\mathrm{STLS}}(\gamma)$, which is the lowest order expression in the iterative solution of Eq. (1), starting from the STLS solution. The lowest-order approximation is capable of improving the STLS approach to the ground-state energy remarkably.

The LFC calculated in the VS approximation has qualitatively similar behavior as $G_{\text {STLS }}(\gamma)$, but has a smaller magnitude. The weak coupling limit of $G_{\mathrm{VS}}(\gamma)$ is $G_{\mathrm{VS}}(\gamma \rightarrow 0) \approx 1 / 2+\left(3 / 2 \pi^{2}\right) \gamma(1-a)$ which reduces to the result given by Gold [8], $G_{\text {STLS }}(\gamma \rightarrow 0) \approx 1 / 2+\left(3 / 2 \pi^{2}\right) \gamma$, as $a \rightarrow 0$. In the Vashishta-Singwi theory the parameter $a$ is determined by adjusting the compressibility calculated using the ground-state energy and that obtained from the long-wavelength limit of the dielectric function. In this work we take $a=2 / 3$ which gives the best agreement with the exact ground state energy. We discuss the compressibility sum-rule in the subsequent sections.

\section{Ground state energy}

The interaction energy (per particle) of a many particle system is written as

$E_{\text {int }}(\gamma)=(1 / 2) \sum_{q} V_{q}\left[N \delta_{q=0}+S(q)-1\right]$,

in which the Hartree contribution is also included. 
Within the mean-field theory this reduces to

$$
E_{\text {int }}(\gamma)=\left(n^{2} / 2 m\right) \gamma\left[1-\frac{1+8 \gamma[1-G(\gamma)] / \pi^{2}}{2\left[1+4 \gamma[1-G(\gamma)] / \pi^{2}\right]^{1 / 2}}\right] \text {. }
$$

The contribution of the interaction energy to the ground state energy per particle is calculated by a coupling constant integration

$E_{\mathrm{int}}=\int_{0}^{\gamma} d \lambda E_{\mathrm{int}}(\lambda) / \lambda$.

The contribution of the kinetic energy to the ground state energy per particle is given by $\epsilon_{\mathrm{F}} / 3=\left(\pi^{2} / 12\right) n^{2} / 2 m$. We express $E_{0}$ in terms of the dimensionless quantity given by

$$
\begin{aligned}
\epsilon(\gamma)= & \frac{\pi^{2}}{12} \\
& +\int_{0}^{\gamma} d \lambda\left[1-\frac{1+8 \lambda[1-G(\lambda)] / \pi^{2}}{2\left[1+4 \lambda[1-G(\lambda)] / \pi^{2}\right]^{1 / 2}}\right],
\end{aligned}
$$

where $E_{0}=\left(n^{2} / 2 m\right) \epsilon(\gamma)$. The series expansion of $\epsilon_{\mathrm{STLS}}(\gamma)$ as $\gamma \rightarrow 0$ is

$$
\begin{aligned}
\epsilon_{\mathrm{STLS}}(\gamma \rightarrow 0)= & \frac{\pi^{2}}{12}+\frac{1}{2} \gamma-\frac{3}{4 \pi^{2}} \gamma^{2}+\frac{5}{12 \pi^{4}} \gamma^{3} \\
& -\frac{7}{16 \pi^{6}} \gamma^{4}+\frac{9}{16 \pi^{8}} \gamma^{5}-\frac{77}{96 \pi^{10}} \gamma^{6} \\
& +\ldots
\end{aligned}
$$

whereas the series expansion of $\epsilon_{\mathrm{VS}}(\gamma)$ in the same limit is

$$
\begin{aligned}
\epsilon_{\mathrm{VS}}(\gamma \rightarrow 0)= & \frac{\pi^{2}}{12}+\frac{1}{2} \gamma-\frac{3}{4 \pi^{2}} \gamma^{2}+\frac{11}{12 \pi^{4}} \gamma^{3} \\
& -\frac{3}{4 \pi^{6}} \gamma^{4}+\frac{19}{48 \pi^{8}} \gamma^{5}-\frac{191}{144 \pi^{10}} \gamma^{6} \\
& +\ldots
\end{aligned}
$$

We note that $\epsilon_{\mathrm{VS}}(\gamma)$ and $\epsilon_{\mathrm{STLS}}(\gamma)$ start to differ at the cubic term in $\gamma$. Fig. 1 shows that the VS approach adopted here gives a better agreement than the STLS result to the exact ground-state energy $\epsilon(\gamma)$. Thus, the validity range of the STLS approximation [8-10] $(\gamma \lesssim 2)$ is increased $(\gamma \lesssim 10)$ within the VS

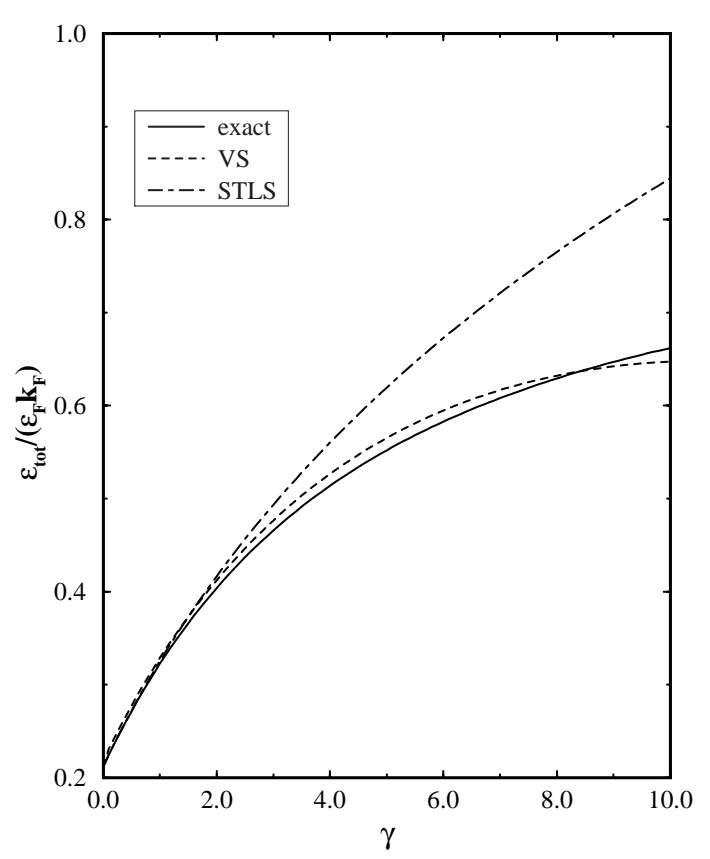

Fig. 1. The ground-state energy per particle $\epsilon(\gamma)$, as a function of the coupling strength $\gamma$. The dot-dashed and solid lines are for the STLS approximation and the exact result of [7], respectively. The dashed line represents the VS approximation for $a=2 / 3$.

approach. Some other ground state quantities of interest are the chemical potential $\mu=\partial E_{0} / \partial N=\left(n^{2} / 2 m\right)(3 \epsilon-\gamma d \epsilon / d \gamma)$, the average potential energy per particle $\langle V\rangle=\left(n^{2} / 2 m\right) \gamma d \epsilon / d \gamma$, and the average kinetic energy $\langle T\rangle=E_{0}-\langle V\rangle=$ $\left(n^{2} / 2 m\right)(\epsilon-\gamma d \epsilon / d \gamma)$. Using the numerically calculated $\epsilon_{\mathrm{VS}}(\gamma)$ we compare these quantities with the results of the exact solution to the 1D fermion problem in Fig. 2. We first note that the STLS approximation results (dotted lines) start deviating from the exact calculation of $\langle T\rangle$ and $\langle V\rangle$ for $\gamma \approx 2$. The VS calculation represents $\langle T\rangle$ reasonably well, but the potential energy term starts to deviate from the exact result for $\gamma \geqslant 6$. However a cancelation effect renders the total energy in quantitative agreement with the exact result up to $\gamma \approx 10$ (see Fig. 1). In the available range of $\gamma$ both the STLS and VS approximations agree well with the exact result for the chemical potential $\mu(\gamma)$. 


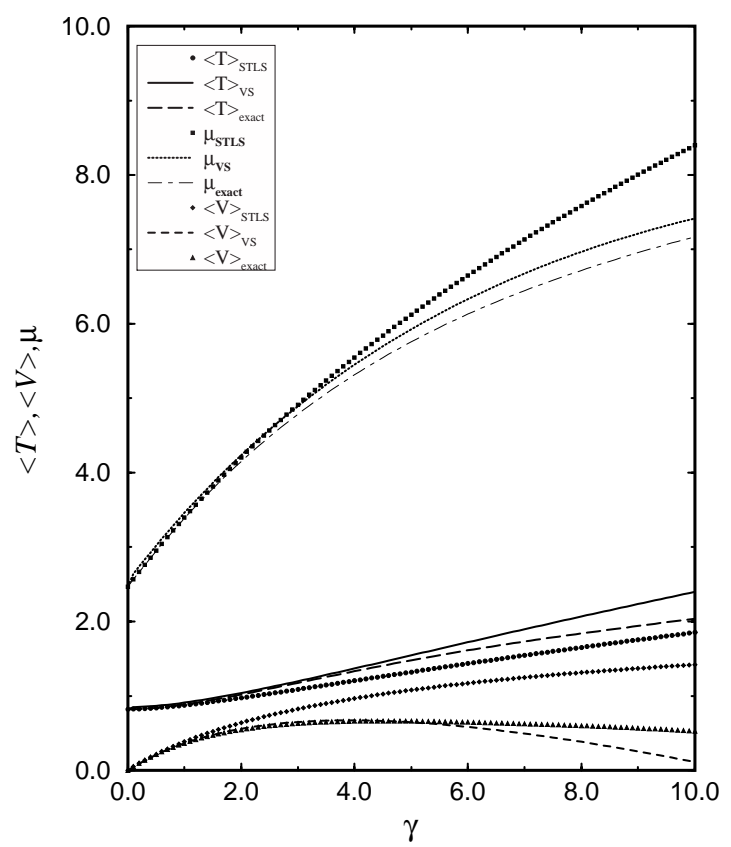

Fig. 2. The chemical potential, average kinetic and potential energies as functions of $\gamma$.

\section{Compressibility}

There are two ways to calculate the compressibility. Firstly, we can calculate the compressibility from the thermodynamic relation

$$
\frac{1}{\kappa}=n^{2} \frac{d^{2}}{d n^{2}}\left(n E_{g}\right) \text {. }
$$

Secondly, we can calculate the compressibility by using the formula $\lim _{q \rightarrow 0} \chi(q, 0)=-n^{2} \kappa$, where the density-density correlation function $\chi(q, \omega)$ of an interacting electron gas with local field correction factor $G(\gamma)$ is given by

$\chi(q, \omega)=\frac{\chi_{0}(q, \omega)}{1-2 n \gamma[1-G(\gamma)] \chi_{0}(q, \omega)}$,

in which $\chi_{0}(q, \omega)$ is the response function of a noninteracting system. Using the thermodynamic relation we find

$$
\frac{\kappa}{\kappa_{0}}=\frac{\pi^{2}}{12 \epsilon(\gamma)-8 \gamma \dot{\epsilon}(\gamma)+2 \gamma^{2} \ddot{\epsilon}(\gamma)},
$$

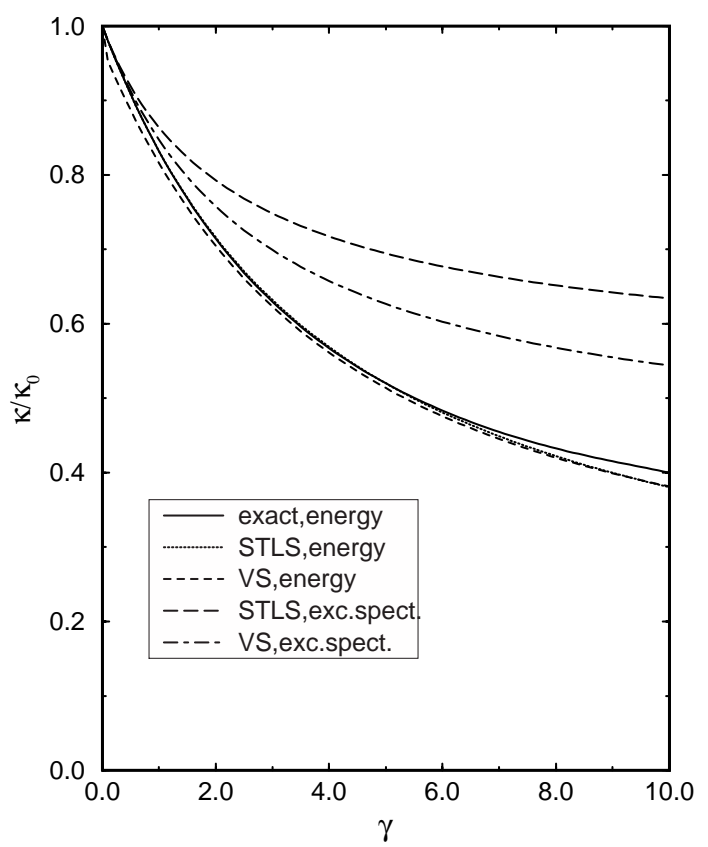

Fig. 3. The compressibility $\kappa$ as a function of $\gamma$. The upper and lower curves are calculated from the excitation spectrum and thermodynamic definition, respectively. The thick solid line is the result using the exact ground-state energy.

whereas the long-wavelength limit of the static density susceptibility yields

$$
\frac{\kappa}{\kappa_{0}}=\frac{\pi^{2}}{\pi^{2}+4 \gamma-4 \gamma G(\gamma)},
$$

where $\kappa_{0}=2 /\left(n^{3} \pi^{2}\right)$ is the compressibility of the noninteracting electron gas in 1D. In Fig. 3 we show the compressibility calculated in the VS and STLS approximations using the above mentioned two different ways. The ground-state energy based calculation of $\kappa$ within the STLS and VS approximations are quite close to the exact result for $\gamma \lesssim 10$. The ground state energy based calculation of $\kappa$ remains below the excitation energy spectrum based calculation of $\kappa$. The VS approach improves the compressibility sum-rule over the STLS result, but does not fulfill it exactly. It is possible to obtain a better agreement by adjusting the parameter $a$, at the expense of worsening the agreement in the ground state energy. 


\section{Pair-correlation function}

The probability of finding two electrons at a distance $r$ is described by the pair-correlation function $g(r)$ which is the Fourier transform of $S(q)$. Performing the one-dimensional Fourier integral analytically [17] we obtain the pair-correlation function within the present model as

$$
\begin{aligned}
g(r)= & g(0)+\gamma^{1 / 2}(1-G)^{1 / 2}\left[I_{1}\left(2 r n \gamma^{1 / 2}(1-G)^{1 / 2}\right)\right. \\
& \left.-\boldsymbol{L}_{1}\left(2 r n \gamma^{1 / 2}(1-G)^{1 / 2}\right)\right] \\
& -\frac{\pi}{\left(2\left[\pi^{2}+4 \gamma[1-G]\right]^{1 / 2}\right)} \\
& +\frac{[n \pi r \sin (n \pi r)+\cos (n \pi r)-1]}{\pi n^{2} r^{2}\left[\pi^{2}+4 \gamma[1-G]\right]^{1 / 2}} \\
& +\frac{\left[\pi^{2}+4 \gamma[1-G]\right]^{1 / 2}}{\pi}-\frac{2 \gamma^{1 / 2}[1-G]^{1 / 2}}{\pi} \\
& -\frac{1}{n \pi r} \int_{0}^{n \pi r} d \tilde{q} \frac{\tilde{q} \cos (\tilde{q})}{\left[\tilde{q}^{2}+4 n^{2} r^{2} \gamma[a 1-G]\right]^{1 / 2}},
\end{aligned}
$$

where $I_{1}(x)$ is the modified Bessel function (of the first kind), $\boldsymbol{L}_{1}(x)$ is the modified Struve function, and $g_{\mathrm{VS}}(0)=1-(2 / \pi) \gamma^{1 / 2}\left(1-G_{\mathrm{VS}}\right)^{1 / 2}$ is the pair-correlation function at zero separation. Fig. 4 compares $g_{\mathrm{VS}}(0)$ with $g_{\mathrm{STLS}}(0)$ as a function of $\gamma$. It was noted that in the STLS approximation $g(0)$ remains positive for all $\gamma$, unlike the Coulomb systems which yield unphysically negative $g(0)$ at some intermediate coupling strength. In the case of Vashishta-Singwi approximation, we find that $g(0)$ eventually becomes negative for $\gamma \gtrsim 10$. Since the theories involving the local-field factor are perturbational in character, thus limiting their applicability for small and intermediate range of $\gamma$, our result for $g_{\mathrm{VS}}(0)$ should be useful in practical applications. The weak coupling limit of $g_{\mathrm{VS}}(0)$ is given by

$$
\begin{aligned}
\lim _{\gamma \rightarrow 0} g_{\mathrm{VS}}(0) \simeq & \frac{1}{2}-\frac{3}{2 \pi^{2}} \gamma+\frac{11}{4 \pi^{4}} \gamma^{2}-\frac{3}{\pi^{6}} \gamma^{3} \\
& +\frac{95}{48 \pi^{8}} \gamma^{4}+\frac{191}{24 \pi^{10}} \gamma^{5}+\cdots
\end{aligned}
$$

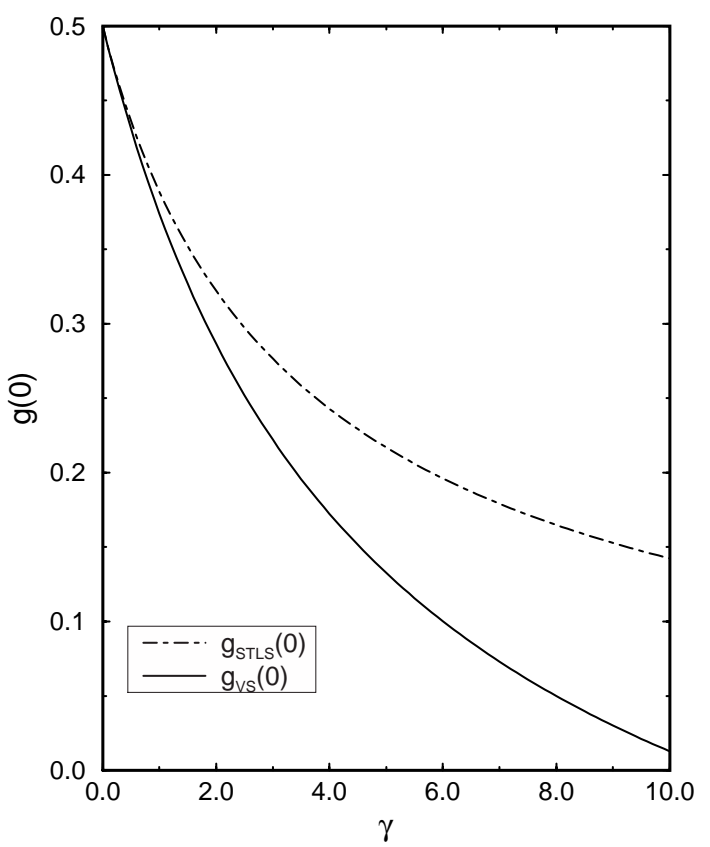

Fig. 4. The pair-correlation function at zero separation $g(0)$ as a function of $\gamma$. The solid and dot-dashed lines indicate $g_{\mathrm{VS}}(0)$, $g_{\text {STLS }}(0)$, respectively.

In the same way, the weak coupling limit of $g_{\mathrm{STLS}}(0)$ is given by

$$
\begin{aligned}
\lim _{\gamma \rightarrow 0} g_{\text {STLS }}(0) \simeq & \frac{1}{2}-\frac{3}{2 \pi^{2}} \gamma+\frac{5}{4 \pi^{4}} \gamma^{2}-\frac{7}{4 \pi^{6}} \gamma^{3} \\
& +\frac{45}{16 \pi^{8}} \gamma^{4}-\frac{77}{16 \pi^{10}} \gamma^{5}+\cdots .
\end{aligned}
$$

The effect of the STLS and VS local-field corrections on the pair-correlation function is further illustrated in Fig. 5 where $g(r)$ is plotted for $\gamma=1$ and $\gamma=5$, and we also specialize to the $a=2 / 3$ case. The differences occur largely at small separations.

\section{Discussion}

In this work we have employed the formalism of Vashishta and Singwi [11] to study the ground state properties of a system of $1 \mathrm{D}$ electron system interacting via a repulsive $\delta$ function potential. In comparison to the STLS approach, the VS local-field description extends the validity range of approximate theories to $\gamma \lesssim 10$, since for this region the ground-state energy 


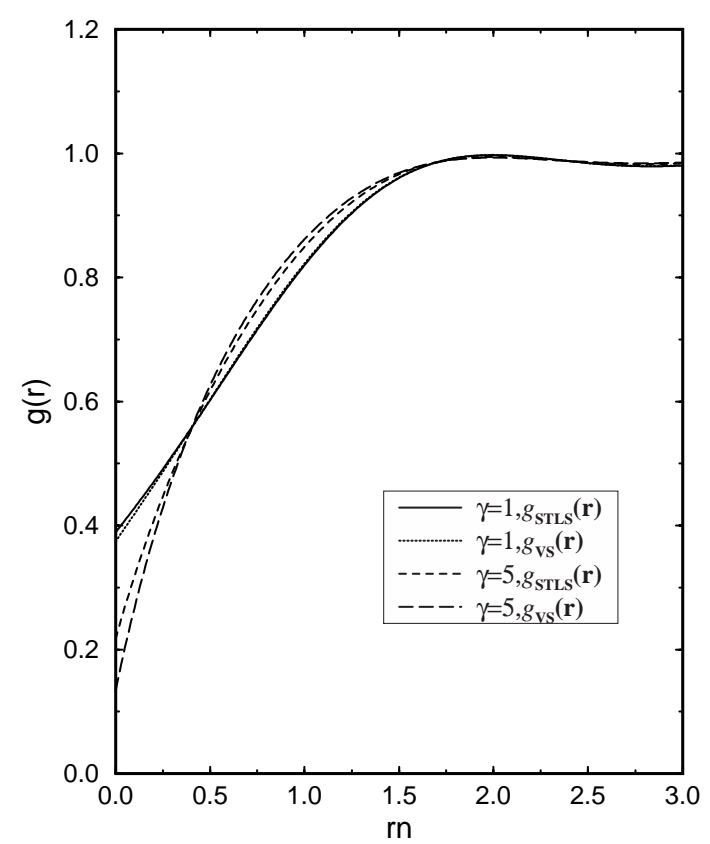

Fig. 5. The pair-correlation function $g(r)$ at $\gamma=1$ and $\gamma=5$ as a function of $r$.

is faithfully reproduced. The compressibility sum-rule is also improved in the VS approach. The strong coupling regime, viz. $\gamma \rightarrow \infty$ is not described well by the STLS and VS approaches, thus we have omitted any discussion on this regime. In the dielectric formulation of the interacting quantum systems the sum-rule constraints [18] on the frequency and wave vector dependent dielectric function $\varepsilon(q, \omega)$ is often found useful. We point out that the frequency and wave vector independent local-field corrections satisfy the first and third moment sum-rules simultaneously. This follows from the fact that the local-field factor is independent of wave vector variable $q$.

\section{Acknowledgements}

This work was partially supported by the Scientific and Technical Research Council of Turkey (TUBITAK) under Grant No. TBAG-1662.

\section{References}

[1] J. Gonzalez, M.A. Martin-Delgado, G. Sierra, A.H. Vozmediano (Eds.), Quantum Electron Liquids and High- $T_{c}$ Superconductivity, Springer, Berlin, 1995.

[2] D.C. Mattis (Ed.), The Many-body Problem, World Scientific, Singapore, 1993.

[3] P. Schlottmann, Int. J. Mod. Phys. B, 11 (1997) 355.

[4] D. Pines, P. Nozieres, The Theory of Quantum Liquids, Benjamin, New York, 1966.

[5] K.S. Singwi, M.P. Tosi, R.H. Land, A. Sjölander, Phys. Rev., 176 (1968) 589.

[6] C.N. Yang, Phys. Rev. Lett., 19 (1967) 1312.

[7] W.I. Friesen, B. Bergersen, J. Phys. C, 13 (1980) 6627.

[8] A. Gold, Phys. Rev. B, 55 (1997) 9470.

[9] A. Gold, J. Phys. Condens. Matter, 10 (1998) 3959.

[10] A. Gold, J. Phys. Condens. Matter, 10 (1998) 3547.

[11] P. Vashishta, K.S. Singwi, Phys. Rev. B, 6 (1972) 875.

[12] E. Demirel, B. Tanatar, unpublished.

[13] N. Iwamoto, D. Pines, Phys. Rev. B, 29 (1991) 3924.

[14] N. Iwamoto, E. Krotscheck, D. Pines, Phys. Rev. B, 29 (1984) 3936.

[15] R.F. Bishop, K.H. Luhrmann, Phys. Rev. B, 26 (1982) 5523.

[16] A. Gold, L. Calmels, Phys. Rev. B, 48 (1993) 11622.

[17] I.S. Gradshteyn, I.M. Rhyzik, Table of Integrals, Series and Products, Academic Press, New York, 1980.

[18] N. Iwamoto, Phys. Rev. A, 30 (1984) 3289. 